Purpose: In this research, anti-inflammatory effect of Stachys Fruticulosa-M.Bieb. extract was studied in rats. The effect of the extract against acute inflammation was studied by hind paw edema test. Methods: Intraperitoneal injection of different doses $(50,100$, 200 and $300 \mathrm{mg} / \mathrm{kg}$ ) of Stachys Fruticulosa which was followed by hind paw carageenan injection $1 \mathrm{~h}$ later were investigated. The resultant edema was quantified by measuring the hind paw edema with three ways of measurement: micrometer method, paw circumference (PC), plethysmometer method. After 2, 4 and $6 \mathrm{~h}$ we measured the anti-inflammatory effect of Stachys Fruticulosa extract. Results and conclusion: Stachys Fruticulosa extract with doses of 100,200 and $300 \mathrm{mg} / \mathrm{kg}$ and indomethacin at a dose of $5 \mathrm{mg} / \mathrm{kg}$ showed significant effect. It is concluded that the methanolic extract of Stachys Fruticulosa-M.Bieb. has anti-inflammatory effect which can be used as an alternative for Indomethacin for treatment of inflammation. Also it might be advantageous to use this herbal drug as a synergist for Indomethacin anti-inflammatory effects, to lower the dosage and consequently complications of NSAIDs.

doi:10.1016/j.toxlet.2012.03.744

\section{P34-03 \\ Formaldeyde exposure during animal macroscopic examination}

Susana Viegas

\section{ESTeSL-IPL, Portugal}

Results of research work developed in anatomy and pathology laboratories have indicated that "macroscopic examination" is the task involving the highest exposure to formaldehyde. This is probably because precision and very good visibility are needed and, therefore, pathologists must lean over the specimen with consequent increase of proximity. With this research we aimed to know formaldehyde exposure in case of animal's macroscopic examination.

Three macroscopic examinations were considered and exposure assessment performed with photo ionization detection (PID) direct-reading equipment (with an $11.7 \mathrm{eV}$ lamp) designated by First-Check, from Ion Science. Higher values of formaldehyde concentration (ceiling values) were register in each exam.

In two of the exams the values obtained were higher (1.3 and $6.1 \mathrm{ppm})$ than the limit-value (ACGIH) for ceiling concentrations $(0.3 \mathrm{ppm})$. The third exam was performed differently, with the protection of macroscopy table down. This situation permitted higher efficacy of exhaustion and avoid the lean over the specimen and, consequently, ceiling value obtain was lower $(0.2 \mathrm{ppm})$.

The importance of this lies in the fact that health effects (cancer) linked to formaldehyde exposure seems to be more related with peaks of high concentrations than with long time exposure at low levels. Furthermore, high exposures of short duration, like those occurring in macroscopic exams, are of special concern, because they can produce an elevated dose rate at target tissues and organs, potentially altering metabolism, overloading protective and repair mechanisms and amplifying tissue responses.Keywords: Formaldehyde; Occupational exposure; Macroscopic exams

doi:10.1016/j.toxlet.2012.03.745

\section{P34-04}

Occupational exposure to toxigenic fungi from Aspergillus flavus complex

Joana Malta-Vacas $^{1}$, Raquel Sabino ${ }^{2}$, Susana Viegas ${ }^{1}$, Carla Viegas ${ }^{1}$

${ }^{1}$ ESTeSL-IPL, Portugal, ${ }^{2}$ DDI-INSA, Portugal

Bioaerosols are mainly composed of fungal particles, bacteria and plant spores, being fungi responsible for the release of VOCs and micotoxins into indoor environments. Aspergillus flavus is a common opportunistic pathogen causing human infections and is involved in the production of aflatoxin and other secondary metabolites associated with toxic and allergic reactions. Poultry workers are exposed to high concentrations of fungi and are therefore more prone to develop associated pathologies.

To evaluate occupational exposure of the workers to Aspergillus flavus and aflatoxins, six animal production facilities were selected, including 10 buildings, from which indoor air samples and outdoor reference samples were obtained.

Twenty-five duplicate samples were collected by two methodologies: impactation onto malt extract agar of 25L air samples using a Millipore Air Tester were used to evaluate quantitative $\left(\mathrm{CFU} / \mathrm{m}^{3}\right)$ and qualitative (species identification, whenever possible) sample composition; $300 \mathrm{~L}$ air samples collected with the Coriolis $\mu$ Air Sampler into phosphate-saline buffer were used to isolate DNA, following molecular identification of Aspergillus section flavi using nor-1 specific primers by real-time PCR.

Overall, Aspergillus was the most frequent genus detected. Using conventional methodologies, A. flavus species were identified in five indoor samples belonging to three buildings and in two outdoor samples. Using real-time PCR, aflatoxigenic species were detected in two buildings, although only one was coincident with the ones identified by cultures.

Using both methodologies we could quantify viable microorganisms and simultaneously identify potentially toxigenic species, resulting in complementary information useful in the adoption of strategies to minimize exposure to micotoxins.

doi:10.1016/j.toxlet.2012.03.746

\section{P34-05}

\section{Futility of travel restriction on air quality: The importance of} training citizens

\author{
Giuliano Polichetti ${ }^{1}$, Alessandra De Sio $^{2}$ \\ ${ }^{1}$ University of Naples “Federico II", Italy, ${ }^{2}$ University of Salerno, Italy
}

Purpose: The preliminary results obtained from studies in Naples and Milan on travel restriction seem to highlight the uselessness of these measures since the concentration of pollutants in the day with or without travel restriction is not statistically significant. Probably this is due to the fact that the government measures do not include the active involvement of citizens and are often a substitute for proper training to an alternative lifestyle. Methods: We made a review of communication and educational activities of government bodies responsible for the health of citizen finding great deficiency. Results and conclusions of the study: Since positive results were obtained from studies that have related the educational programs for the adoption of awareness lifestyles with a better asthma care and greater understanding of the factors by which increase the risks (such as ambient air pollution), we believe that training and information remain the main tools available to governments for the 